\title{
AN INEQUALITY FOR GENERALIZED QUADRANGLES
}

\author{
STANLEY E. PAYNE
}

\begin{abstract}
Let $\delta$ be a generalized quadrangle of order $(s, t)$. Let $X$ and $Y$ be disjoint sets of pairwise noncollinear points of $\mathcal{S}$ such that each point of $X$ is collinear with each point of $Y$. If $m=|X|$ and $n=|Y|$, then $(m-1)(n$ $-1)<s^{2}$. When equality holds, severe restrictions are placed on $m, n, s$, and $t$.
\end{abstract}

I. Prolegomena. A generalized quadrangle of $\operatorname{order}(s, t), s \geqslant 1, t \geqslant 1$, is a point-line incidence geometry $\mathcal{S}=(\mathscr{P}, \mathcal{L}, I)$ with point set $\mathscr{P}$, line set $\mathcal{L}$, and symmetric point-line incidence relation $I$ satisfying the following axioms:

A1. No two points are incident with two lines in common.

A2. If $x$ is a point not incident with a line $L$, then there is a unique point $y$ incident with $L$ and collinear with $x$.

A3. Each line (respectively, point) is incident with $1+s$ points (respectively, $1+t$ lines).

Throughout this note $\mathcal{S}=(\mathscr{P}, \mathcal{L}, I)$ will denote a generalized quadrangle (GQ) of order $(s, t), s \geqslant 1, t \geqslant 1$. Let $X=\left\{x_{1}, \ldots, x_{m}\right\}$ and $Y=$ $\left\{y_{1}, \ldots, y_{n}\right\}$ be disjoint sets of pairwise noncollinear points of $\mathcal{\delta}, m \geqslant 2$ and $n \geqslant 2$. Let $k_{i}$ be the number of $x_{j}$ 's with which $y_{i}$ is collinear, $1 \leqslant i \leqslant n$, $0 \leqslant k_{i} \leqslant m$. Our main results consist of the following two theorems.

THEOREM I.1.

$$
(1+s) \cdot \sum_{i=1}^{n} k_{i} \leqslant m n+\sqrt{m^{2} n^{2}+\left(s^{2}-1\right)(m+n) m n+\left(s^{2}-1\right)^{2} m n .}
$$

THEOREM I.2. Let $k_{i}=m$ for all $i$, i.e. each $y_{i}$ is collinear with each $x_{j}$. Then $(m-1)(n-1) \leqslant s^{2}$. If equality holds, then one of the following must occur.

(i) $m=n=1+s$, and each point of $Z=\mathscr{P} \backslash(X \cup Y)$ is collinear with precisely two points of $X \cup Y$.

(ii) $m \neq n$. If $m<n$, then $s \mid t, s<t, n=1+t, m=1+s^{2} / t$, and each point of $\mathcal{S}$ is collinear with either 1 or $1+t / s$ points of $Y$ according as it is or is not collinear with some point of $X$. Note: $(m-1) \mid s$.

There are two corollaries that deserve mention.

COROLlaRY I.3. If there is a GQ $\mathcal{\text { with }}$ order $(s, t), s>1$, then $t \leqslant s^{2}$. If $t=s^{2}$, then each triad of points has exactly $1+s$ centers.

Received by the editors February 14, 1977.

AMS (MOS) subject classifications (1970). Primary 05B25; Secondary 15A18.

Key words and phrases. Generalized quadrangles, Rayleigh quotient, incidence matrix. 
Proof. The inequality $t \leqslant s^{2}$ is due to D. G. Higman ([3], [4]). Alternate treatments appear in Bose [1] and Cameron [2]. In the present setting a proof is obtained by putting $X=\left\{x_{1}, x_{2}\right\}$ where $x_{1}$ and $x_{2}$ are not collinear, $Y=\operatorname{tr}(X)=$ the set of $1+t$ points collinear with both $x_{1}$ and $x_{2}$, and then applying Theorem I.2.

COROllaRY I.4. Let $x$ and $y$ be noncollinear points of $\mathcal{S}$ with $s>1$ and $|\operatorname{sp}(x, y)|=1+p$. Then $p t \leqslant s^{2}$. If $p t=s^{2}$ and $p<t$, then each point $z$ collinear with no point of $\operatorname{sp}(x, y)$ must be collinear with exactly $1+t / s$ points of $\operatorname{tr}(x, y)$.

Proof. For the original proof and an explanation of the notation see Thas [7]. In the present setting put $X=\operatorname{sp}(x, y), Y=\operatorname{tr}(x, y)$.

The proofs depend on a general matrix theoretic approach due to Sims. As the treatment in [5] does not include the "case of equality," we first give an exposition of this method.

II. A matrix-theoretic technique. If $\bar{x}=\left(x_{1}, \ldots, x_{n}\right)^{T}$ and $\bar{y}=$ $\left(y_{1}, \ldots, y_{n}\right)^{T}$ are column vectors of real numbers, then $\bar{x} \cdot \bar{y}=\sum x_{i} y_{i}$ denotes their usual dot product. If $A$ is a real, symmetric, $n \times n$ matrix, then for each $\bar{x} \neq \overline{0}$ define the Rayleigh quotient $R(\bar{x})$ for $A$ by

$$
R(\bar{x})=\frac{\bar{x} \cdot A \bar{x}}{\bar{x} \cdot \bar{x}}
$$

It is well known that $A$ has real characteristic roots, say $\mu_{1} \leqslant \cdots \leqslant \mu_{n}$, and that

$$
\mu_{1}=\min _{\bar{x}: \bar{x} \neq \overline{0}} R(\bar{x}) \leqslant \max _{\bar{x}: \bar{x} \neq \overline{0}} R(\bar{x})=\mu_{n} .
$$

Perhaps not so well known is the following.

II.1. Let $\bar{x}$ be a nonzero vector in $R^{n}$ for which $R(\bar{x})=\mu_{i}$ for either $i=1$ or $i=n$. Then $\bar{x}$ is a characteristic vector of $A$ belonging to the characteristic value $\mu_{i}$.

Proof. Let $\bar{x}_{1}, \ldots, \bar{x}_{n}$ be an orthonormal basis of characteristic vectors of $A$ ordered so that $A \bar{x}_{i}=\mu_{i} \bar{x}_{i}$. Let $\bar{x}$ be an arbitrary nonzero vector of $R^{n}$ normalized so that $\bar{x} \cdot \bar{x}=1$. Then $R(\bar{x})=\bar{x} \cdot A \bar{x}$ and $\bar{x}=\sum c_{i} \bar{x}_{i}$ with $\sum c_{i}^{2}$ $=1$. Hence $\mu_{1}=\mu_{1} \cdot \sum c_{i}^{2} \leqslant \sum c_{i}^{2} \mu_{i}=\bar{x} \cdot A \bar{x}=R(\bar{x})$, with equality holding if and only if $\mu_{i}=\mu_{1}$ whenever $c_{i} \neq 0$. It follows that $R(\bar{x})=\mu_{1}$ if and only if $\bar{x}$ belongs to the eigenspace associated with $\mu_{1}$. The argument for $\mu_{n}$ is similar.

We continue to let $A=\left(a_{i j}\right)$ denote an $n \times n$ real symmetric matrix. Let $\Delta=\Delta_{1}+\cdots+\Delta_{r}$ and $\Gamma=\Gamma_{1}+\cdots+\Gamma_{s}$ be partitions of $\{1, \ldots, n\}$. Suppose that $\Gamma$ is a refinement of $\Delta$, and write $i \subseteq j$ whenever $\Gamma_{i} \subseteq \Delta_{j}$, $1 \leqslant i \leqslant s, 1 \leqslant j \leqslant r$. Put $\delta_{i}=\left|\Delta_{i}\right|, \gamma_{i}=\left|\Gamma_{i}\right|$. Let

$$
\delta_{i j}=\sum_{\substack{\mu \in \Delta_{i} \\ \gamma \in \Delta_{j}}} a_{\mu \nu} ; \gamma_{i j}=\sum_{\substack{\mu \in \Gamma_{i} \\ \nu \in \Gamma_{j}}} a_{\mu \nu} .
$$


So $\delta_{i j}=\delta_{j i}$ and $\gamma_{i j}=\gamma_{j i}$ by the symmetry of $A$. Define the following matrices:

$$
\begin{aligned}
& A^{\Delta}=\left(\frac{\delta_{i j}}{\delta_{i}}\right)_{1<i, j<r} ; \quad A^{\Gamma}=\left(\frac{\gamma_{i j}}{\gamma_{i}}\right)_{1<i, j<s} . \\
& \overline{A_{\Delta}}=\operatorname{diag}\left(\sqrt{\delta_{1}}, \ldots, \sqrt{\delta_{r}}\right) ; \quad \bar{A}_{\Gamma}=\operatorname{diag}\left(\sqrt{\gamma_{1}}, \ldots, \sqrt{\gamma_{s}}\right) . \\
& \hat{A}_{\Delta}=\bar{A}_{\Delta} A^{\Delta}\left(\overline{A_{\Delta}}\right)^{-1}=\left(\frac{\delta_{i j}}{\sqrt{\delta_{i} \delta_{j}}}\right)_{1<i, j<r} . \\
& \hat{A}_{\Gamma}=\bar{A}_{\Gamma} A^{\Gamma}\left(\overline{A_{\Gamma}}\right)^{-1}=\left(\frac{\gamma_{i j}}{\sqrt{\gamma_{i} \gamma_{j}}}\right)_{1<i, j<s} .
\end{aligned}
$$

Hence $\hat{A}_{\Delta}$ and $\hat{A}_{\Gamma}$ are real symmetric matrices with real characteristic values equal to those of $A^{\Delta}$ and $A^{\Gamma}$, respectively. The smallest and largest characteristic roots of $\hat{A}_{\Gamma}$ and $\hat{A}_{\Delta}$ are the minimum and maximum, respectively, of $\left(\bar{x} \cdot \hat{A}_{\Gamma} \bar{x}\right) /(\bar{x} \cdot \bar{x})$ and $\left(\bar{y} \cdot \hat{A}_{\Delta} \bar{y}\right) /(\bar{y} \cdot \bar{y}), \overline{0} \neq \bar{x} \in R^{s}, \overline{0} \neq \bar{y} \in R^{r}$.

Let $0 \neq \bar{y}=\left(y_{1}, \ldots, y_{r}\right)^{T} \in R^{r}$. Then put $\bar{x}=\left(\ldots, x_{\alpha}, \ldots\right)^{T}$, where $x_{\alpha}=y_{i} \sqrt{\gamma_{\alpha} / \delta_{i}}$ whenever $\alpha \subseteq i, 1 \leqslant \alpha \leqslant s$. Then

$$
\sum_{\alpha=1}^{s} x_{\alpha}^{2}=\sum_{i=1}^{r}\left(\sum_{\alpha \subseteq i}\left(y_{i} \sqrt{\gamma_{\alpha} / \gamma_{i}}\right)^{2}\right)=\sum_{i=1}^{r} \frac{y_{i}^{2}}{\delta_{i}}\left(\sum_{\alpha \subseteq i} \gamma_{\alpha}\right)=\sum_{i=1}^{r} y_{i}^{2},
$$

implying $\bar{x} \cdot \bar{x}=\bar{y} \cdot \bar{y}$. And

$$
\begin{aligned}
\bar{x} \cdot \hat{A}_{\Gamma} \bar{x} & =\sum_{\alpha, \beta=1}^{s} x_{\alpha} \frac{\gamma_{\alpha \beta}}{\sqrt{\gamma_{\alpha} \gamma_{\beta}}} x_{\beta} \\
& =\sum_{i, j=1}^{r}\left(\sum_{\substack{\alpha \subseteq i \\
\beta \subseteq j}} \frac{\gamma_{\alpha \beta}}{\sqrt{\gamma_{\alpha} \gamma_{\beta}}} \cdot \frac{y_{i} \sqrt{\gamma_{\alpha}}}{\sqrt{\delta_{i}}} \cdot \frac{y_{j} \sqrt{\gamma_{\beta}}}{\sqrt{\delta_{j}}}\right) \\
& =\sum_{i, j=1}^{r} y_{i}\left(\sum_{\substack{\alpha \subseteq i \\
\beta \subseteq j}} \frac{\gamma_{\alpha \beta}}{\sqrt{\delta_{i} \delta_{j}}}\right) y_{j} \\
& =\sum_{i, j=1}^{r} y_{i}\left(\frac{\delta_{i j}}{\sqrt{\delta_{i} \delta_{j}}}\right) y_{j}=\bar{y} \cdot \hat{A}_{\Delta} \bar{y} .
\end{aligned}
$$

This implies that any value of $\left(\bar{y} \cdot \hat{A}_{\Delta} \bar{y}\right) /(\bar{y} \cdot \bar{y})$ is also a value of $\left(\bar{x} \cdot \hat{A}_{\Gamma} \bar{x}\right) /(\bar{x}$ $\cdot \bar{x})$. Hence the following is a corollary of (2) and II.1.

II.2. If $\mu_{1} \leqslant \cdots \leqslant \mu_{r}$ are the characteristic roots of $A^{\Delta}$ and $\lambda_{1} \leqslant \cdots \leqslant$ $\lambda_{s}$ are the characteristic roots of $A^{\Gamma}$, then $\lambda_{1} \leqslant \mu_{1} \leqslant \mu_{r} \leqslant \lambda_{s}$. If $\bar{y}=$ $\left(y_{1}, \ldots, y_{r}\right)^{T}$ satisfies $A A^{4}=\lambda_{1} y\left(\right.$ so $\left.\lambda_{1}=\mu_{1}\right)$, then $A^{\Gamma} \bar{x}=\lambda_{1} \bar{x}$, where $\bar{x}=$ $\left(\ldots, x_{\alpha}, \ldots\right)^{T}$ is defined by $x_{\alpha}=y_{i}$ whenever $\alpha \subseteq i$. A similar result holds in case $\lambda_{n}=\mu_{n}$. 
Proof. The first part of the result is evident. So let $\overline{0} \neq \bar{y}=\left(y_{1}, \ldots, y_{r}\right)^{T}$ satisfy $A A^{4} \bar{y}=\lambda_{1} \bar{y}=\mu_{1} \bar{y}$. Then $\overline{A_{\Delta}} \bar{y}=\left(y_{1} \sqrt{\delta_{1}}, \ldots, y_{r} \sqrt{\delta_{r}}\right)^{T}$ is a characteristic vector of $\hat{A}_{\Delta}$ belonging to $\lambda_{1}=\mu_{1}$. Hence $\bar{z}=\left(\ldots, z_{\alpha}, \ldots\right)^{T}$, $z_{\alpha}=y_{i} \sqrt{\gamma_{\alpha}}$ for $\alpha \subseteq i$, is a characteristic vector of $\hat{A}_{\Gamma}$ belonging to $\lambda_{1}$ (by the proof of II.1). It follows that $\bar{x}$ as given in the statement of II.2 is a characteristic vector of $A^{\Gamma}$ associated with $\lambda_{1}$. A similar proof holds in case $\lambda_{n}=\mu_{n}$.

III. Applications to generalized quadrangles. Let $\delta=(\mathscr{P}, \mathcal{L}, I)$ be a GQ of order $(s, t)$. Let $X$ and $Y$ be as in the hypothesis of Theorem I.1, and put $Z=\mathscr{P} \backslash(X \cup Y)$, so $|Z|=r=v-(m+n)$, where $v=(1+s)(1+s t)=$ $|\mathscr{P}|$. For some ordering of $\mathscr{P}$ let $A$ be the $(0,1)$-matrix $A=\left(a_{i j}\right)$ defined by $a_{i j}=1$ if the $i$ th and $j$ th points of $\mathscr{P}$ are not collinear in $\mathcal{S} ; a_{i j}=0$ otherwise. It follows that $A$ is symmetric with minimum polynomial given by $f(x)=(x$ $+s)(x-t)\left(x-t s^{2}\right)$. Let $\Delta=\Delta_{1}+\Delta_{2}+\Delta_{3}$ be the partition of $\{1, \ldots, v\}$ determined by $X, Y$, and $Z$; i.e. points of $X, Y, Z$, respectively, are indexed by $\Delta_{1}, \Delta_{2}, \Delta_{3}$, respectively. As $\delta_{i}=\left|\Delta_{i}\right|$, we have $\delta_{1}=m, \delta_{2}=n, \delta_{3}=v-(m$ $+n), \delta_{11}=n(n-1), \delta_{12}=\delta_{21}=\sum_{i=1}^{n}\left(m-k_{i}\right) m n=\Sigma$, where $\Sigma=\sum_{i=1}^{n} k_{i}$. Since $\Sigma_{j=1}^{3}\left(\delta_{i j} / \delta_{i}\right)=t s^{2}$, we also have $\delta_{13}=\delta_{1} t s^{2}-\delta_{12}-\delta_{11}=t s^{2} m-(m n$ $-\Sigma)-m(m-1)$. Similarly, $\delta_{23}=t s^{2} n-(m n-\Sigma)-n(n-1)$. Using these results it is now routine to complete the calculation of $A^{\Delta}$.

$$
A^{\Delta}=\left(\begin{array}{ccc}
m-1 & n-\Sigma / m & t s^{2}+1-m-n+\Sigma / m \\
m-\Sigma / n & n-1 & t s^{2}+1-m-n+\Sigma / n \\
A_{1} & A_{2} & A_{3}
\end{array}\right)
$$

where

$$
A_{1}=\frac{m\left[t s^{2}+1-m-n\right]+\Sigma}{v-m-n}, \quad A_{2}=\frac{n\left[t s^{2}+1-m-n\right]+\Sigma}{v-m-n}
$$

and

$$
A_{3}=t s^{2}-\frac{(m+n)\left[t s^{2}+1-m-n\right]+2 \Sigma}{v-m-n} .
$$

Let $\left(x-t s^{2}\right)\left(x-r_{1}\right)\left(x-r_{2}\right)$ be the characteristic polynomial of $A^{\Delta}$ with the roots ordered so that $r_{1} \leqslant r_{2} \leqslant t s^{2}$. Let $\Gamma=\Gamma_{1}+\cdots+\Gamma_{v}$ be the identity partition of $\{1, \ldots, v\}$, so $\Gamma$ is a refinement of $\Delta$. Then $A^{\Gamma}=A$ has numerical range $\left[-s, t s^{2}\right]$ which must then contain all characteristic roots of $A^{\Delta}$. Indeed, the proof of Theorem I.1 amounts to calculating $r_{1}$ and using the inequality $-s \leqslant r_{1}$. We now proceed to do this.

Put $\left(x-r_{1}\right)\left(x-r_{2}\right)=x^{2}-b x+c$, so that $2 r_{1}=b-\sqrt{b^{2}-r c}$. Hence $-s \leqslant r_{1}$ simplifies to

$$
0 \leqslant s^{2}+b s+c, \quad b=r_{1}+r_{2}=\operatorname{tr}\left(A^{\Delta}\right)-t s^{2}, \quad c=\operatorname{det}\left(A^{\Delta}\right) / t s^{2} .
$$

It is easy to calculate $\operatorname{tr}\left(A^{\Delta}\right)$ from (3) and then to write $b$ as follows. 


$$
b=\frac{(m+n)(s+s t+2)-2 v-2 \Sigma}{v-m-n} .
$$

To calculate $\operatorname{det}\left(A^{\Delta}\right)$, add the first and second columns of $A^{\Delta}$ to the third column and then subtract the first row from the second. At this point $\operatorname{det}\left(A^{\Delta}\right)$ appears as follows.

$$
\operatorname{det}\left(A^{\Delta}\right)=t s^{2}\left|\begin{array}{lll}
m-1 & n-\Sigma / m & 1 \\
1-\Sigma / n & \Sigma / m-1 & 0 \\
\frac{m\left[t s^{2}+1-m-n\right]+\Sigma}{v-m-n} & \frac{n\left[t s^{2}+1-m-n\right]+\Sigma}{v-m-n} & 1
\end{array}\right| .
$$

Expanding by the third column and simplifying, one may calculate $c$ to be as follows.

$$
c=\operatorname{det}\left(A^{\Delta}\right) / t s^{2}=\frac{(1+s+s t)(2 \Sigma-m-n)+v-v \Sigma^{2} / m n}{v-m-n} .
$$

Using the values for $b$ and $c$ given in (5) and (7), (4) may be rewritten as follows.

$$
0 \leqslant(s-1)\left(m+n+s^{2}-1\right) m n+2 m n \Sigma-(1+s) \Sigma^{2} .
$$

Equality in (8) gives two roots $\Sigma_{1}$ and $\Sigma_{2}$ for which (8) says $\Sigma_{1} \leqslant \Sigma \leqslant \Sigma_{2}$, if $\Sigma_{1} \leqslant \Sigma_{2}$. But $\Sigma_{2}$ is easily evaluated.

$$
\Sigma_{2}=\frac{m n+\sqrt{m^{2} n^{2}+\left(s^{2}-1\right)(m+n) m n+\left(s^{2}-1\right)^{2} m n}}{1+s} .
$$

Clearly $\Sigma \leqslant \Sigma_{2}$ is just the inequality in Theorem I.1. If each $k_{i}=m$, then $\Sigma=m n$, and the inequality of Theorem I.1 reduces to $(m-1)(m-1) \leqslant s^{2}$, the inequality of Theorem I.2.

We now use II.2 to investigate the case of equality in Theorem I.2. Suppose that $k_{i}=m$ for all $i$, so $\Sigma=m n$, and suppose that $(m-1)(n-1)=s^{2}$, so $-s$ is a characteristic root of $A^{\Delta}$. Hence a nonzero characteristic vector of $A^{\Delta}$ belonging to $-s$ must span the null space of $A^{\Delta}+s I$.

$$
A^{\Delta}+s I=\left(\begin{array}{ccc}
m-1+s & 0 & t s^{2}+1-m \\
0 & n-1+s & t s^{2}+1-n \\
* & * & *
\end{array}\right)
$$

where we need not bother to calculate the third row, since the rank must equal 2. Clearly $\bar{y}=\left(y_{1}, y_{2}, 1\right)^{T}$ spans the null space of $A^{\Delta}+s I$, where

$$
y_{1}=\frac{m-1-t s^{2}}{s+m-1} ; \quad y_{2}=\frac{n-1-t s^{2}}{s+n-1} .
$$

Let us assume that the points of $\mathscr{P}$ are ordered (for the construction of $\mathrm{A}$ ) so that the first $m$ points are those of $X$, the next $n$ points are those of $Y$, and the last $v-m-n$ points are those of $Z$. Then by II.2, $\bar{x}$ must be a characteristic vector of $A^{\Gamma}=A$ belonging to $\lambda_{1}=-s$, where $\bar{x}$ is as follows. 


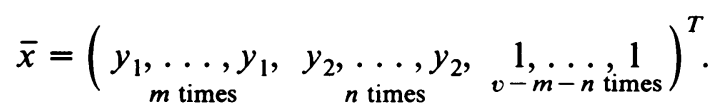

For the first $m+n$ rows of $A$ this yields no new information. But let $z \in Z$ be the $i$ th point, $i>m+n$. Suppose $z$ is not collinear with $t_{1}$ points of $X$, is not collinear with $t_{2}$ points of $Y$, and hence is not collinear with $t s^{2}-t_{1}-t_{2}$ points of $Z$. Then the product of the $i$ th row of $A$ with $\bar{x}$, which must equal $-s$, is actually $t_{1} y_{1}+t_{2} y_{2}+t s^{2}-t_{1}-t_{2}=s$. After a little simplification this becomes

$$
\frac{t_{1}}{s+m-1}+\frac{t_{2}}{s+n-1}=1
$$

If $z$ lies on a line joining a point of $X$ and a point of $Y$, then $t_{1}=m-1$ and $t_{2}=n-1$, i.e., since $\delta$ has no triangles, $z$ is collinear with a unique point of $X$ and with a unique point of $Y$. On the other hand, if $z$ is not on such a line either $t_{1}=m$ or $t_{2}=n$. Suppose $t_{1}=m$, so $z$ is collinear with no point $X$. Using (13) we find that the number of points of $Y$ collinear with $z$ is

$$
n-t_{2}=1+(n-1) / s \text {. }
$$

Similarly, any point of $\mathscr{P}$ collinear with no point of $Y$ must be collinear with $1+(m-1) / s$ points of $X$. If $m=n=s+1$, this says each point not on a line joining a point of $X$ with a point of $Y$ must be collinear with two points of $X$ and none of $Y$ or with two of $Y$ and none of $X$. If $1<m<s+$ 1 , so $1+(m-1) / s$ is not an integer, then each point of $\mathscr{P}$ is collinear with some point of $Y$. This implies that each point $z$ of $Z$ is either on a line joining points of $X$ and $Y$ or is collinear with $1+(n-1) / s \geqslant 3$ points of $Y$. Clearly $n \leqslant 1+t$. Suppose $n<1+t$ and let $x_{1} \in X$. Then there is some line $L$ through $x_{1}$ not incident with any point of $Y$. But then any point $z$ on $L$, $z \neq x_{1}$, cannot be collinear with any point of $Y$, a contradiction. Hence it must be that $n=1+t$, from which it follows that $m=1+s^{2} / t$. This essentially completes the proof of Theorem I.2.

A similar treatment is available for the restriction on the parameters of a subquadrangle, a combinatorial proof of which is found in [6].

\section{REFERENCES}

1. R. C. Bose, Geometric and pseudo-geometric graphs $\left(q^{2}+1, q+1,1\right)$, J. Geometry 2 (1972), 75-93.

2. P. J. Cameron, Partial quadrangles, Quart. J. Math. Oxford Ser. 25 (1974), 1-13.

3. D. G. Higman, Partial geometries, generalized quadrangles, and strongly regular graphs, Atti Convegno di Geometria e sue Applicazioni, Perugia, 1971.

4. Invariant relations, coherent configurations, and generalized polygons, Combinatorics, Part 3, Math. Centre Tracts \# 57, Amsterdam, 1974, $27-43$.

5. S. E. Payne, Finite generalized quadrangles: a survey, Proc. Internat. Conf. Proj. Planes, Washington State Univ. Press, 1973, 219-261.

6. J. A. Thas, 4 remark concerning the restriction on the parameters of a 4 -gonal subconfiguration, Simon Stevin 48 (1974-75), 65-68.

7. $\longrightarrow$ On generalized quadrangles with parameters $s=q^{2}$ and $t=q^{3}$, Geometria Dedicata 5 (1976), 485-496.

Department of Mathematics, Miami University, Oxford, Oho 45056 